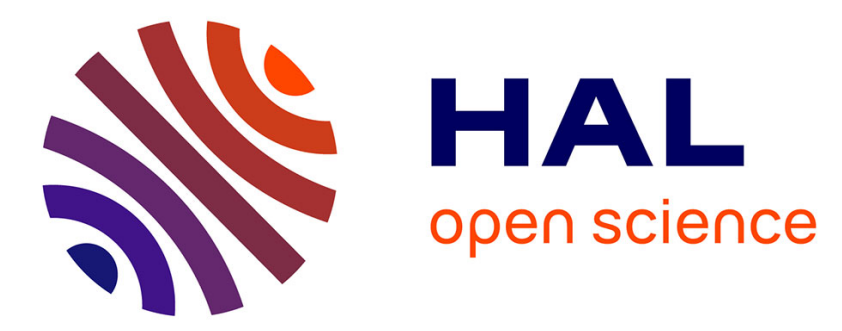

\title{
The a.c. susceptibility third harmonic component of NdO1-0.14F0.14FeAs: A flux dynamic magnetic analysis
}

Daniele Di Gioacchino, Augusto Marcelli, Alessandro Puri, Antonio Bianconi

\section{To cite this version:}

Daniele Di Gioacchino, Augusto Marcelli, Alessandro Puri, Antonio Bianconi. The a.c. susceptibility third harmonic component of NdO1-0.14F0.14FeAs: A flux dynamic magnetic analysis. Journal of Physics and Chemistry of Solids, 2010, 71 (8), pp.1046. 10.1016/j.jpcs.2010.03.004 . hal-00661917

\section{HAL Id: hal-00661917 https://hal.science/hal-00661917}

Submitted on 21 Jan 2012

HAL is a multi-disciplinary open access archive for the deposit and dissemination of scientific research documents, whether they are published or not. The documents may come from teaching and research institutions in France or abroad, or from public or private research centers.
L'archive ouverte pluridisciplinaire HAL, est destinée au dépôt et à la diffusion de documents scientifiques de niveau recherche, publiés ou non, émanant des établissements d'enseignement et de recherche français ou étrangers, des laboratoires publics ou privés. 


\section{Author's Accepted Manuscript}

The a.c. susceptibility third harmonic component of NdO1-0.14F0.14FeAs: A flux dynamic magnetic analysis

Daniele Di Gioacchino, Augusto Marcelli, Alessandro Puri, Antonio Bianconi

PII: S0022-3697(10)00050-8

DOI: doi:10.1016/j.jpcs.2010.03.004

Reference: PCS 6113

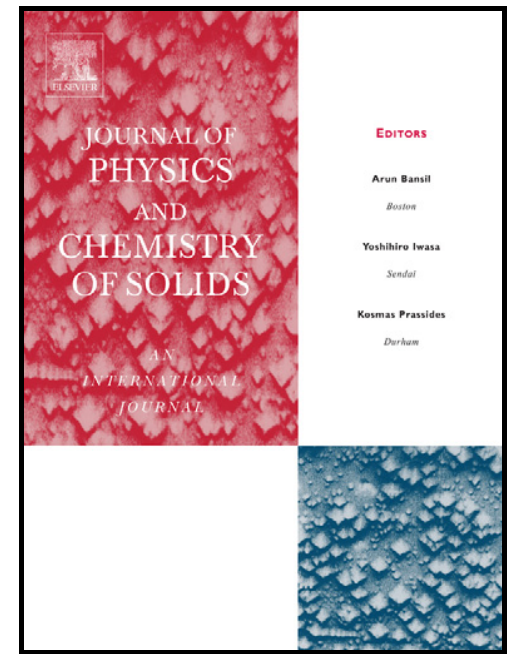

www.elsevier.com/locate/jpcs

To appear in: Journal of Physics and Chemistry of Solids

Cite this article as: Daniele Di Gioacchino, Augusto Marcelli, Alessandro Puri and Antonio Bianconi, The a.c. susceptibility third harmonic component of NdO1-0.14F0.14FeAs: A flux dynamic magnetic analysis, Journal of Physics and Chemistry of Solids, doi:10.1016/j.jpcs.2010.03.004

This is a PDF file of an unedited manuscript that has been accepted for publication. As a service to our customers we are providing this early version of the manuscript. The manuscript will undergo copyediting, typesetting, and review of the resulting galley proof before it is published in its final citable form. Please note that during the production process errors may be discovered which could affect the content, and all legal disclaimers that apply to the journal pertain. 


\title{
The a.c. susceptibility third harmonic component of $\mathrm{NdO}_{1-0.14} \mathrm{~F}_{0.14} \mathrm{FeAs}$ : a flux dynamic magnetic analysis
}

\author{
Daniele Di Gioacchino ${ }^{\mathrm{a}}$, Augusto Marcelli ${ }^{\mathrm{a}}$, Alessandro Puri ${ }^{\mathrm{a}, \mathrm{b}}$ and Antonio Bianconi ${ }^{\mathrm{b}}$ \\ ${ }^{a}$ INFN - LNF, Via E. Fermi, 40 - 00044 Frascati (Rome), Italy \\ ${ }^{b}$ University 'La Sapienza', Dipartimento di Fisica, P.le A. Moro, 4 - 00100 Rome, Italy
}

\begin{abstract}
.
In this contribution we present the analysis of the third harmonic susceptibility data of the new superconductor $\mathrm{NdFeAs}_{1-0.14} \mathrm{~F}_{0.14}$. 'Cole-Cole' polar plots respect to the magnetic frequency of the exciting field are presented and discussed. Data show that $\mathrm{NdFe} \mathrm{As}_{1-0.14} \mathrm{~F}_{0.14}$ exhibits a 'bulk pinning' with a 3D flux dynamic character. A comparison of the responses of high $T_{c}$ materials and an evaluation of theoretical critical states is also presented.
\end{abstract}

Phase transition, High harmonic magnetic ac susceptibility measurements, $\mathrm{NdO}_{1-0.14} \mathrm{~F}_{0.14} \mathrm{FeAs}$.

\section{INTRODUCTION}

The recent discovery of superconductivity in arsenide oxide materials [1] triggered many different investigations on these materials. $\mathrm{RFeAsO}_{1-\mathrm{x}} \mathrm{F}_{\mathrm{x}}$ systems $\left(\mathrm{R}=\right.$ rare earth) are characterized by a high $\mathrm{T}_{\mathrm{c}}$ (from 26 to $\sim 55 \mathrm{~K}$ ) and a layered crystal structure with FeAs layers associated to the superconductivity and $\mathrm{RO}_{1 . \mathrm{x}} \mathrm{F}_{\mathrm{X}}$ layers acting as charge reservoirs. In these compounds a complex interplay among magnetism, layered structure and multiple surface Fermi sheets occur [2-4]. In addition, low magnetic field and high-field transports measurements [5-6] both show an intermediate behavior between that of a low- $\mathrm{T}_{\mathrm{c}}$ superconductor and HTSC cuprates. In this contribution we present a flux dynamic investigation, to obtain information on the thermal fluctuation of vortices and the dimensionality of the pinning processes connected to the critical current behavior vs. temperature, applied magnetic field in the sample. Actually these parameters are important for practical applications and the ac multi-harmonic susceptibility [7], in particular the third harmonic component [8] is the best tool for this kind of analysis. In addition to the a.c. third harmonic study of the $\mathrm{NdO}_{1}$. ${ }_{0.14} \mathrm{~F}_{0.14} \mathrm{FeAs}$ as a function of both temperature and frequencies in a low applied magnetic field, we compare the observed behaviors with ideal critical state models and with those of other high $\mathrm{T}_{\mathrm{c}}$ materials (HTSC) such as $\mathrm{MgB}_{2}, \mathrm{YBCO}$ and $\mathrm{BiSCOO} 2223$.

\section{A.C. MULTI HARMONIC SUSCEPTIBILITY AND FLUX DYNAMIC IN A SUPERCONDUCTOR}

The dissipative effects in the superconductor mixed flux vortex state, can be investigated with "ac magnetic susceptibility" (X) experiments [7]. In Fig. 1 we show the real part of the first harmonic $\left(X^{\prime}{ }_{1}\right)$ (red curve) vs.

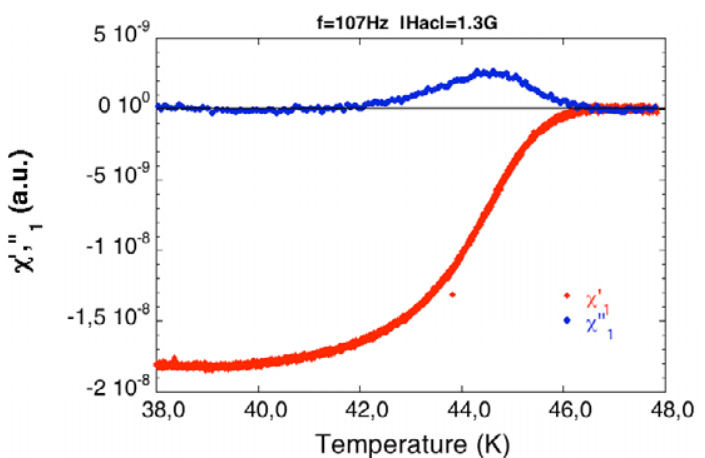

Fig. 1. ac susceptibility measurements vs. temperature of the $\mathrm{NdFeAsO} \mathrm{O}_{1-0.14} \mathrm{~F}_{0.14}$ sample. The real part of the first harmonic (red curve) and the imaginary part (blue curve).

temperature for one sample of $\mathrm{NdO}_{1-0.14} \mathrm{~F}_{0.14} \mathrm{FeAs}$. The curve describes the magnetic behavior of a characteristic superconductor sample with a pure negative diamagnetic signal. At the same time as the first harmonic imaginary part $\left(X{ }_{1}\right)$ (blue curve) is correlated to the magnetic energy absorbed by both linear and non-linear processes, and is proportional to the magnetization (M) cycle vs a.c. applied field $\left(\mathrm{H}_{\mathrm{ac}}\right)$ [9]. The interpretation of the harmonic susceptibility in a superconductor is based on critical-state models [10-12] where the magnetic B profile is correlated, by the Ampere's law, to the current induced in the sample that is equal to the critical value $\mathrm{I}_{\mathrm{c}}$. Indeed, in a one-dimensional sample with a thickness $2 \mathrm{~d}$, the width $\Delta$ inside a sample where is induced a supercurrent $\mathrm{I}_{\mathrm{c}}$, is $\Delta=\mathrm{B}_{\mathrm{ac}} / \mathrm{I}_{\mathrm{c}}$, where $\mathrm{B}_{\mathrm{ac}}=\mu_{0} \mathrm{H}_{\mathrm{ac}}$. As a consequence, when $\Delta=\mathrm{d}$ a supercurrent flows through the entire sample volume. In this case the internal local field $B_{p}$ can be written as $B^{*}=I_{c} d$ and the first and higher harmonic susceptibilities depends only by one parameter: $\delta=B_{p} / B_{a c}$ [13]. In other words when $\delta=1$, the penetration field reaches the center of the sample.

In particular the low frequency complex multi harmonic susceptibility $\left(\mathrm{X}_{\mathrm{n}}=\mathrm{X}_{\mathrm{n}}^{\prime}+\mathrm{i}_{\mathrm{n}}{ }^{\prime \prime}\right)$ can be used to identify the non-linear processes that sustain the superconducting critical current density and the linear normal losses that limit the superconducting properties. The method offers great advantages because, while skin electronic losses are linear processes and the susceptibility measures only the first harmonic component, a phase transition, in particular a superconducting transition, is a non-linear processes characterized by non-harmonic contributions. In this latter case, high harmonics $\left(X_{n}\right)$ together with the first 
component are simultaneously presents in the susceptibility behaviors.

High harmonic components can be regarded as the Fourier coefficients [13] of the steady magnetization cycles in the presence of an external oscillating magnetic field. These coefficients are affected by the magnetic flux entering and leaving the sample. The hysteretic behavior and the high harmonic susceptibility components describe the interaction between the flux vortex array and extrinsic properties such as the pinning due to the disorder present in the material. However, the analysis is complex due to the large number of parameters involved: the coherence length $(\xi)$ that describes the core of the weak pinning potential in a disordered state; the inter-vortex distance $\left(\mathrm{a}_{0}\right)$ leading to the pinned vortex bundle formation; $\lambda$ defining the flux extension range, the elastic properties of the flux lattice defined by the $\mathrm{C}_{\mathrm{ii}}$ elastic modules such as flux tilt $\left(\mathrm{C}_{44}\right)$, shear $\left(\mathrm{C}_{66}\right)$, compression $\left(\mathrm{C}_{11}\right)$ [14], the mean thermal displacement $\langle u\rangle$ of the flux vortex and the dimensionality ( $\mathrm{nD}$ with $\mathrm{n}=1,2,3$ ) of the flux-pinning interaction due to the material layered structure. The correlations between these scale lengths determine the presence of different flux pinning motion regimes respect to magnetic field, temperature and electric current with a specific vortex mobility described by the characteristic 'time relation' or 'window frequency' occurring in a superconductor, e.g., single vortexes, small bundle vortexes, large bundle vortexes, weak or strong pinning interactions.

In this scenario, decreasing the temperature the vortex system moves in a non-equilibrium critical state that slowly decays toward the equilibrium towards a final flux glass state $[15,16]$.

The susceptibility components $X_{n}$ and in particular the third harmonic susceptibility probes the superconducting electric transport processes in different regions of a 'phenomenological space' [16] whose axis are the magnetic field, the temperature and the current (Fig. 2). Different ' $H_{a c}$ frequencies' induce changes in the current level and may set different 'time windows' for each process. In particular, the high harmonic component of the magnetic susceptibility is related to

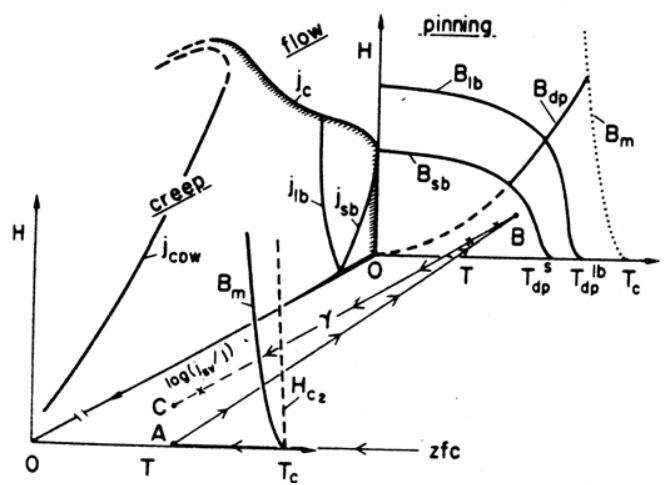

Fig. 2. A phenomenological phase diagram Magnetic fieldTemperature-Current for an anisotropic HTSC material. The axis of the current is proportional to the time [16] the I-V superconductor characteristic. The latter is strongly non-linear and, as showed in Fig. 3, the ' $\mathrm{I}-\mathrm{V}$ curvature' describes the dynamic behavior of flux lattice-pinning interactions around the critical current value $I_{c}$. The $I-V$ curvature and high harmonics $\left(X_{n>1}\right)$ are proportional quantities. When the frequency increases, in a superconductor grows the potential V actually probing changes in the ' $\mathrm{I}-\mathrm{V}$ curvature' associated to the different flux dynamics induced in the sample [17].

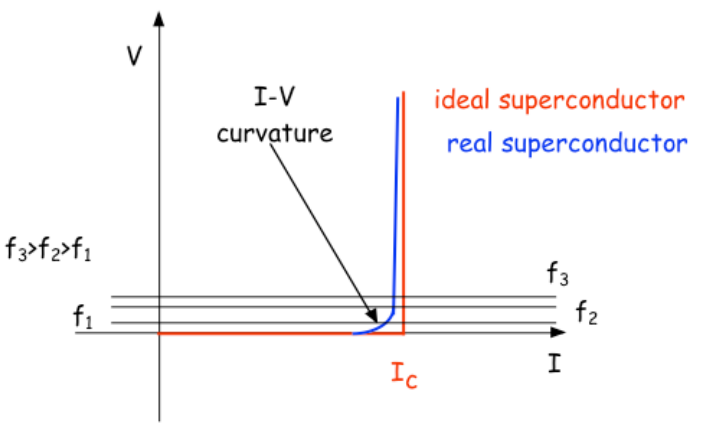

Fig. 3. The I-V superconductor characteristic. Different potential $\mathrm{V}$ are showed for three different frequencies $\left(\mathrm{f}_{1}<\mathrm{f}_{2}<\mathrm{f}_{3}\right)$.

\section{EXPERIMENTAL SET-UP}

The superconducting $\mathrm{NdF}_{0.14} \mathrm{FeAsO}_{1-0.14}$ bulk sample has been prepared by high pressure synthesis from $\mathrm{Nd}$, As, $\mathrm{Fe}, \mathrm{Fe}_{2} \mathrm{O}_{3}, \mathrm{FeF}_{3}$ powders (the purity of all starting chemicals was better than $99.99 \%$ ). The components were mixed together according to the nominal stoichiometric ratio of $\mathrm{NdO}_{1-0.14} \mathrm{~F}_{0.14} \mathrm{FeAs}$, then grounded thoroughly and pressed into small pellets. The latter were sealed in boron nitride crucibles and sintered in a high-pressure synthesis apparatus under a pressure of $6 \mathrm{GPa}$ and at the temperature of $1300{ }^{\circ} \mathrm{C}$ for two hours [18]. The sample dimensions are $1.5 \times 1.5 \times 1.0 \mathrm{~mm}$. The ac high harmonics susceptibility response of the sample has been measured in a susceptometer based on pick-up double coils surrounded by a driven coil (Fig.4) [19]. The sample was set on a sapphire holder, which fits in the pick-up coil bridge. Two coils connected in series and rolled up in opposition realize the bridge. One

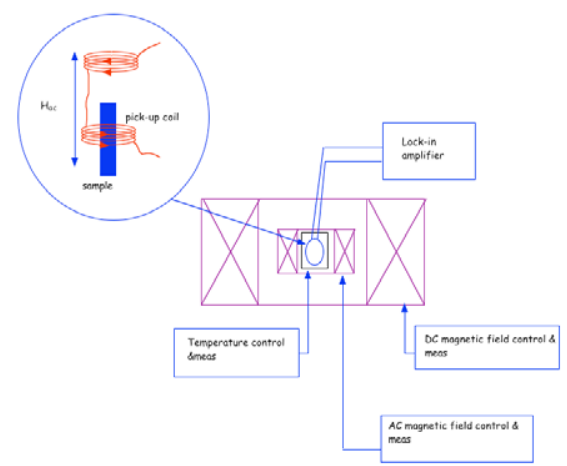

Fig. 4. A schematic layout of the a.c. susceptibility measurements. 
is strongly coupled (pick-up coil) while the second is weakly (balance coil) coupled to the sample. The sample is cooled in the 'Zero magnetic Field Cooling (ZFC) setup' in a thermally controlled He gas flow cryostat. The range of the ac driving magnetic field frequency was $107 \mathrm{~Hz}<\mathrm{f}<1070 \mathrm{~Hz}$ with an amplitude of 1.3 Gauss. A multi harmonic lock-in amplifier measured the Fourier components of the induced signal. The sample was oriented parallel to the ac magnetic field, and during the acquisition the temperature has been measured by a CGR thermometer. Susceptibility data are the result of the subtractions between signals collected with and without the sample vs. frequency and temperature.

\section{IV. $\mathrm{NDO}_{1-0.14} \mathrm{~F}_{0.14} \mathrm{FEAS}$ THIRD HARMONIC MEASUREMENTS AND DISCUSSION}

In Fig. 5 we show the real and imaginary parts $\left(\mathrm{X}_{3}\right.$, $\left.X{ }_{3}\right)$ of the third harmonic of the $\mathrm{NdF}_{0.14} \mathrm{FeAsO}_{1-0.14}$ sample versus temperature and frequency. Real parts describe the oscillating signals around the zero value. All the odd high harmonic susceptibility components are polynomial functions with maxima and minima of the ratio $\delta,[13]$ a parameter proportional to the penetration depth of the ac magnetic applied field into the sample and connected with the critical current via critical state models. For $\delta<1$ odd high harmonic real part vanish. In addition, in this pure sinusoidal excitation case, the even harmonic components disappear [13]. The behaviors
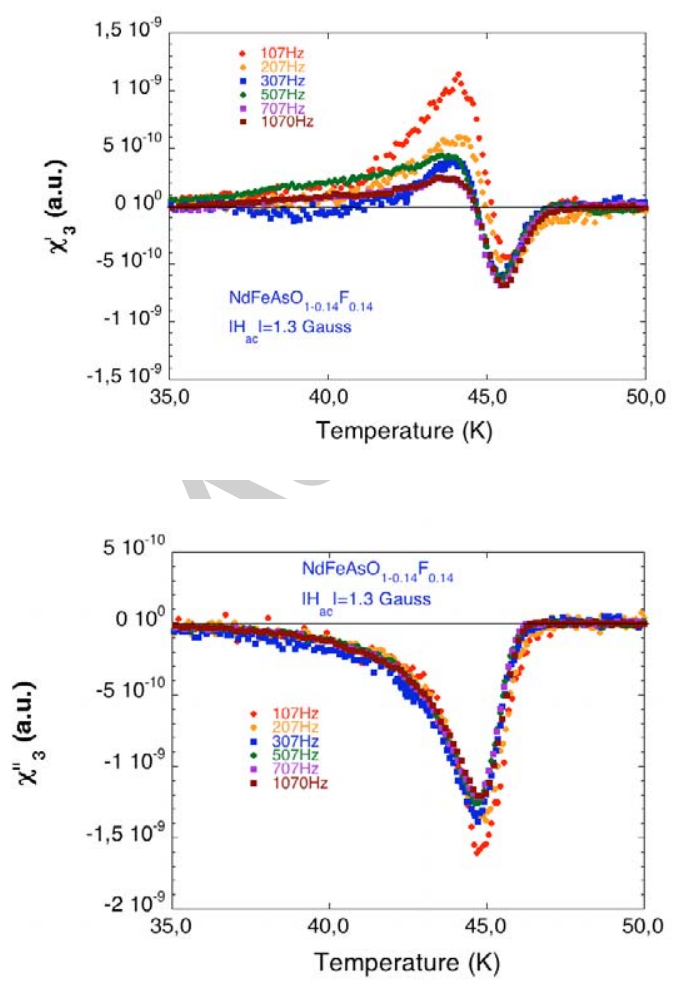

Fig. 5. The third harmonic components $\left(X_{1-3}^{\prime}, X_{1-3}\right)$ of the ac magnetic susceptibility curves vs. temperature and frequency for the $\mathrm{NdFeAsO}_{1-0.4} \mathrm{~F}_{0.4}$ sample. (top: real part; bottom: imaginary part). allow identifying the onset temperature of the sample, i.e., the critical temperature $\mathrm{T}_{\mathrm{c}}$ at $46.5 \mathrm{~K}$.

In a typical ZFC procedure, after cooling a magnetic field is applied to the sample and the superconductor switches in a flux-pinning critical state. The state decays with time, via an 'activated temperature creep' process, in a final stable flux-pinning 'Glass state' pattern (see Fig. 2) [16]. The frequency dependence of the non-linear magnetic response describes this behavior. For this sample, at low magnetic field and around $T_{c}$, the 'critical state' decays in a 'Glass state' with a faster characteristic creep time rate respect to the explored experimental time window range (9.3$0.93 \mathrm{msec}$ ), corresponding to an applied field frequency in the range $107-1070 \mathrm{~Hz}$. For this reason, the $X_{3}{ }_{3}, X^{\prime}{ }_{3}$ temperature onsets are characterized by a very weak frequency dependence.

The frequency dependence of the third harmonic susceptibility components vs. temperature is illustrated in Fig. 5 and describes the change of the effective fluxdiffusivity connected with the flux-pinning interaction. This variation is due to the change of the potential $\mathrm{V}$ induced by the frequency, as described in the superconducting I-V characteristic shows in Fig. 3.

To clarify the role of the $X_{3}$ frequency dependence, we may analyze the polar plot (Cole-Cole) of $X^{\prime}{ }_{3}$ vs. $X^{\prime \prime}{ }_{3}$ components [20-22]. These lens shaped closed curves showed in Fig. 6, address both a change of the initial $X$ phase and of the area of the closed curves as a function of the applied magnetic field frequency. In this figure we show the presence of a 'pure critical state with a homogeneous pinning bulk' almost independent by the frequency with a 'lens' shape [20,21] (red curve) and a “pure critical state with a surface pinning barrier' with a cardioid profile (violet curve) [21].

The first indication of the 'lens shape' closed curves is that a 'flux bulk pinning' is operative in the $\mathrm{NdF}_{0.14} \mathrm{FeAsO}_{1-0.14}$ sample. The second is the rotation of the polar plots in a counter-clockwise mode if compared to the ideal bulk pinning critical state $[13,20,23]$. This corresponds to a change of the 'phase lag' $\Theta$ with the frequency excitation, associated to a temporal shift between the magnetic excitation signal $\left(\mathrm{B}_{\mathrm{ac}}\right)$ on the

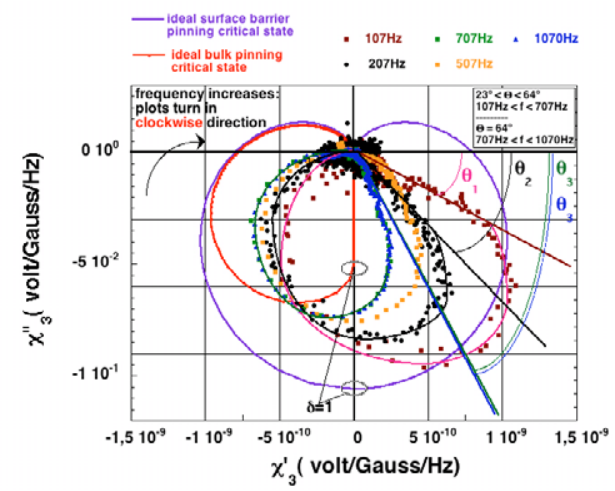

Fig. 6. $\mathrm{NdFeAsO} \mathrm{A}_{1-0.14} \mathrm{~F}_{0.14}$ polar plots (Cole-Cole), i.e., $\mathrm{X}_{3}^{\prime}$ vs. $\mathrm{X}_{3}{ }_{3}$ vs. frequency (temperature is an implicit variable). The ideal 'lens shape bulk pinning' (red) and the 'cardioid shape surface barrier' (violet) critical states are also showed for comparison. 
surface and to the magnetic response induced at some distance below the surface $\left(B_{p}\right)$. The shift is correlated to an effective non-linear magnetic diffusivity due to the flux-pinning interaction [20]. The $\Theta$ frequency behavior showed in Fig. 6 is correlated also to the different I-V curvatures [17] observed in the real case and probed vs. frequency. It depends by the flux motion, the pinning interaction and by its dimensionality inside the sample [17].

All critical state models describe magnetic configuration independent by the frequency. However, due to the 'bulk pinning', in the critical state the $X_{3}^{\prime}-X_{3}{ }_{3}$ Cole-Cole plot is characterized by the phase $\Theta=90^{\circ}$ up to $\delta=1$ because for $\delta \leq 1$ the $X_{3}^{\prime}$ amplitude is equal to zero (see Fig. 6 - red curve) [13] while in the 'surface pinning barrier' this occurs only for $\delta=1$ [21].

Data of the $\mathrm{NdFe} A s \mathrm{O}_{1-0.14} \mathrm{~F}_{0.14}$ show a phase change with a clockwise rotation increasing the frequency in the range $23^{\circ}<\Theta<64^{\circ}$ when the frequency is in the range 107 $-707 \mathrm{~Hz}$. On the contrary, polar plots are independent in the $707-1070 \mathrm{~Hz}$ range although at $\Theta=64^{\circ}$ the value is different by the ideal one in the critical state $\left(\Theta_{\text {Bulk pinning }}=\right.$ $90^{\circ}$ ) probably due to the finite thickness of the sample [23]. Actually, at high frequencies, the magnetic flux profile inside the $\mathrm{NdFeAs} s_{1-0.4} \mathrm{~F}_{0.4}$ sample is substantially similar to the critical state and at these frequencies. The potential $\mathrm{V}$ induced in the sample determines around the $\mathrm{I}_{c}$ value, a similar curvature in the I-V characteristic. In this case experiments probes an I-V characteristic region with a very steep slope, e.g., $f_{2}$ or $f_{3}$ in Fig. 3 .

It is useful for the analysis of the flux dynamic in the $\mathrm{NdF}_{0.14} \mathrm{FeAsO}_{1-0.14}$, the comparison of the third harmonic polar behavior, measured with the same experimental set-up used for others HTSC materials characterized by a different flux-dimensionality, pinning and critical temperature.

In Fig. 7 we compare the $X^{\prime}{ }_{3}-X^{\prime \prime}{ }_{3}$ Cole-Cole plots of three HTSC materials at different frequencies. Here the temperature is considered an implicit variable.

The $\mathrm{MgB}_{2}$ sample (panel $\mathrm{A}$ ) has a $\mathrm{T}_{\mathrm{c}}=38.2 \mathrm{~K}$ and it is characterized by a $X_{3}$ polar plots with a clockwise rotation vs. frequency in the angular range $43^{\circ}<\Theta<74^{\circ}$ and in the interval $107-1070 \mathrm{~Hz}$. The frequency rotation of the plots is similar to that of the $\mathrm{NdFeAsO}_{1-0.14} \mathrm{~F}_{0.14}$ with a clockwise rotation. However, at high frequency is not present a frequency independent region. Other differences with the $\mathrm{NdFeAs} \mathrm{s}_{1-0.4} \mathrm{~F}_{0.4}$ plots are connected with the closed curve contours that have a cardioids-like shape. However, increasing the frequency, the 'cardioid' position of the $\mathrm{MgB}_{2}$ departs from the ideal position of the 'surface barrier flux pinning critical state' showed in Fig. 6 (violet line) where the $\Theta_{\text {barrier surface pinning }}=0^{\circ}$. In this case, a flux dynamic dominated by a three dimensional (3D) Kim-Anderson 'creep activation' in a bulk collective pinning scenario it is also possible [8]. The claiming is based on the numerical solution of the nonlinear magnetic diffusion equation for the magnetic flux motion in a superconductor of thickness $d$ with sinusoidal boundary conditions such as the 'set-up' of a.c. susceptibility experiments:
Panel A

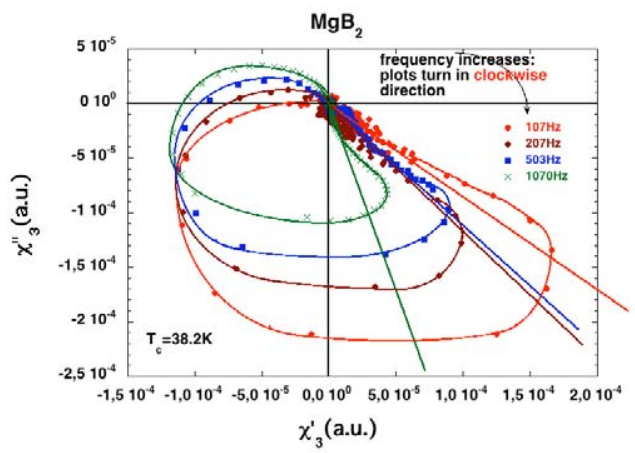

Panel B

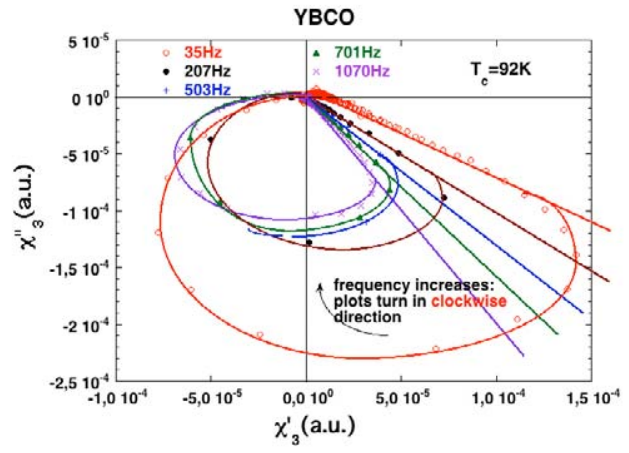

Panel C

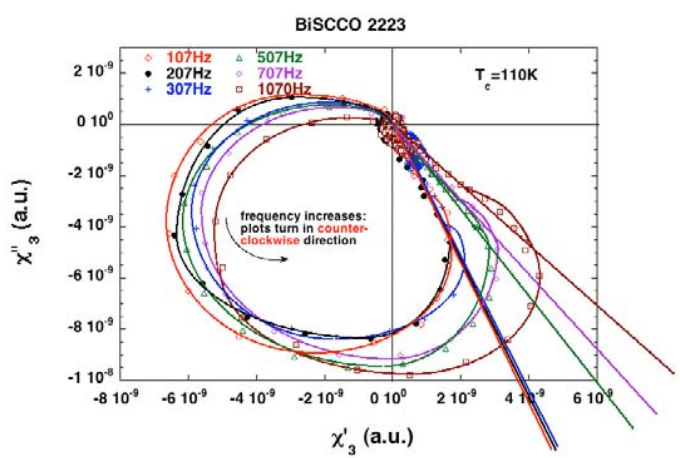

Fig. 7. $\mathrm{MgB}_{2}$ (A), YBCO (B), Bi2223 (C) X polar plots for different frequencies (the temperature is an implicit variable).

$$
\frac{\partial B}{\partial \text { time }}=\frac{\partial}{\partial x} \cdot\left[\left(\frac{\rho(B, J)}{\mu_{0}}\right) \frac{\partial B}{\partial x}\right]
$$

in which the diffusion coefficient $\rho(\mathrm{J})$, is described by the Kim-Anderson creep resistivity expression:

$$
\rho_{\text {creep }}=2 \rho_{f f} \frac{J_{c}(t)}{J} \cdot e^{-U_{p}(t) / K T} \cdot \sinh \left[\frac{J U_{p}(t)}{J_{c}(t) K T}\right]
$$

where $\rho_{\text {ff }}$ is the flux flow resistivity, $t=T / T_{c}$ is the reduced temperature and 


$$
J=\frac{1}{\mu_{0} d} \cdot \frac{\partial B}{\partial \breve{x}}
$$

is the current density induced in the sample where $\breve{x}=x / d$ is a dimensionless variable.

The temperature dependence of the pinning potential and the critical current in the collective pinning model used are [8]:

$U_{p}=U_{0}\left(1-t^{4}\right)$

$J_{c}=J_{c 0}\left(1-t^{2}\right)^{3 / 2}\left(1+t^{2}\right)^{-1}$

The results analyzed using the Cole-Cole $\mathrm{X}_{3}$ plot are showed in Fig. 8. We may recognize a 'cardioid-like' feature and a frequency clockwise rotation. Although the polar plot in the figure shows a different behavior respect to the $\mathrm{MgB}_{2}$, for this numerical case [8] we used the $\mathrm{YBCO}$ parameters with a thickness $\mathrm{d}=1 \mathrm{~cm}$ larger than that of the $\mathrm{MgB}_{2}$ sample.

In a YBCO sample with a $T_{\mathrm{c}}=92 \mathrm{~K}$ the polar plots of the $X_{3}$ in Fig. 7 (panel B) also show an increasing clockwise rotation vs. frequency in the range $30^{\circ}<\Theta<58^{\circ}$ and $35-1070 \mathrm{~Hz}$. All closed curves have an ellipsoidal (lens) characteristic and for this sample both the frequency rotation and the plot look similar to those of the $\mathrm{NdF}_{0.14} \mathrm{FeAsO}_{1-0.14}$. Also in this case an independent behavior of the $\Theta$ angle at high frequency values is not observed in the explored frequency range. The flux dimensionality in the YBCO layered superconductor, has been strongly debated but at present it has been substantially established that a 3D flux pinning behavior occurs for a low amplitude of magnetic field [24].

The $\mathrm{X}_{3}$ of a BiSCCO 2223 tape with $\mathrm{T}_{\mathrm{c}}=110 \mathrm{~K}$ gives the polar plots of Fig. 7 (panel C) with a counterclockwise rotation vs. frequency. The polar plots in the range $107-307 \mathrm{~Hz}$ have the angular value $\Theta=63^{\circ}$ independent by the frequency, while in the $307-1070 \mathrm{~Hz}$ interval the angular value ranges between $63^{\circ}<\Theta<42^{\circ}$.

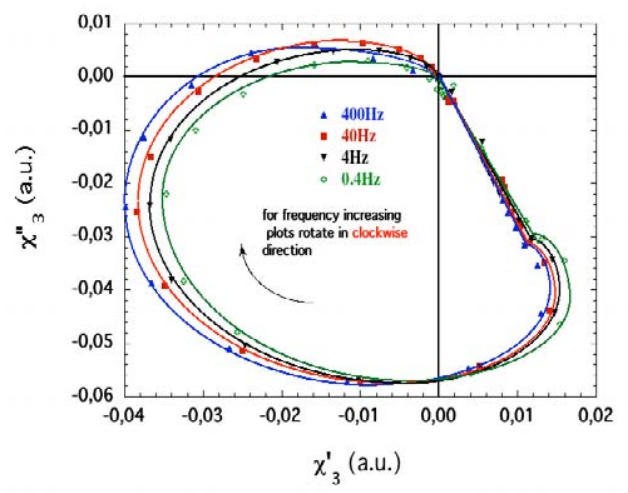

Fig. 8. Polar plots (Cole-Cole) $X_{3}^{\prime}$ vs. $X_{3}{ }_{3}$ results from 0.4 to 400 $\mathrm{Hz}$ (the temperature is an implicit variable) based on the solutions of the non-linear magnetic diffusion equation (Eq. 1) with the creep resistivity (Eq.2) and a collective pinning model (Eq. 3 and 4). See the Fig. 9 in the Ref. [8].
The magnetic flux profile inside the BiSCCO 2223 at low frequencies is quite similar to that in the critical state. The frequency rotation exhibited by the $\mathrm{NdFeAsO}{ }_{1-0.14} \mathrm{~F}_{0.14}$ sample displays the opposite behavior. Another difference in the closed curve profiles, are contours between cardioid/lens features. These behaviors address for the BiSCCO material a clear 2D flux dimensionality [25]. Indeed, respect to the YBCO system, in this layered oxide superconductor material the $\mathrm{CuO}$ metallic planes are strongly separated by buffer layers. As a consequence a large anisotropy is present and an array of 'pancake flux vortices' on the $\mathrm{CuO}$ layer joined by very weak Josephson junctions is a suitable description of the observed behavior. Considering also the high $\mathrm{T}_{\mathrm{c}}$ of the Bi2223 sample, for temperatures around $T_{c}$, Josephson junctions are broken and only separate superconducting layers remain. Only pinning in the $\mathrm{CuO}$ plane is active, and an uncoupled layered material with a clear 2D flux dynamic behavior exists. In this case, only at low frequencies a critical state independent by the frequency occurs. When the frequency increases in a weak 2D flux-pinning material, the potential $\mathrm{V}$ induced in the sample and the thermal fluctuations are able to melt the pinning vortex lattice. As a consequence the superconductor critical state changes in a liquid state [26].

To investigate the 2D behavior, we considered the numerical solutions of the non-linear diffusion equation (Eq.1) with a resistivity coefficient described by the collective pinning $[27,28]$ in the glass model $[17,29]$ with a flux creep dynamic regimes:

$$
\rho_{\text {creep }}=\rho_{f f} \frac{J_{c}(B, T)}{J} \cdot e^{-U_{p}(B, T) / \mu K T \cdot\left[\left(J_{c}(B, T) / J\right)^{\mu}-1\right]}
$$

In this $2 \mathrm{D}$ bulk pinning framework, considering the elastic response of the flux vortex lattice in a superconductor [30] only the shearing $\mathrm{C}_{66}$ elastic moduli [31] is active while the tilt $\mathrm{C}_{44}$ elastic moduli $[17,28]$ is not yet effective:

$U_{p}(B, T) \propto\left(\frac{\xi(B, T)}{a_{0}}\right)^{2} \cdot C_{66}(B, T) \cdot d$

$J_{p}(B, T) \propto C_{66}(B, T) \cdot \xi(B, T)$

The magnetic behaviors of these parameters are $[8,17,28]$ :

$\mathrm{C}_{66}(\mathrm{~B}, \mathrm{~T}) \propto \mathrm{B}_{\mathrm{c} 2}^{2}(\mathrm{t}) \cdot\left[\mathrm{b}\left(1-\mathrm{b}^{2}\right) \cdot\left(1-0.58 \mathrm{~b}+0.29 \mathrm{~b}^{2}\right)\right]$

$\xi(B, T) \propto \sqrt{\left(1+t^{2}\right) /\left(1-t^{2}\right)} / \sqrt{1 /(2-2 b)}$

$a_{0}^{2}=\Phi_{0} / B \quad ; b=B / B_{c 2}(T)$

The results summarized in Fig. 9 confirm that in a 
2D flux pinning bulk state, increasing the frequency the $\mathrm{X}_{3}$ polar plots have a counter-clock rotation behavior.

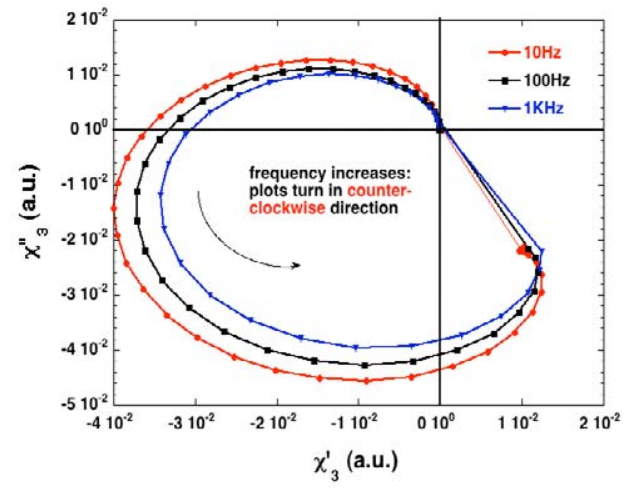

Fig. 9. Polar plots (Cole-Cole) $X_{3}^{\prime}$ vs. $X_{3}{ }_{3}$ data in the frequency range $10-1 \mathrm{KHz}$ (the temperature is a implicit variable). Data are based on the solutions of the non-linear magnetic diffusion equation with the creep resistivity (Eq.2) and the 2D glass collective pinning model (Eq. 5-10).

Moreover for the BiSCCO 2223 sample showed in Fig. 7 (panel $\mathrm{C}$ ) if we consider that the rotation changes the low frequency 'lens shape' into a high frequency 'cardioid-like' feature as a function of the frequency, a shift in the flux dynamic occurs. At high frequency, the superconducting response is induced near the tape surface and a 'surface barrier' pinning is presents. If the 'surface' pinning is stronger respect to the $2 \mathrm{D}$ bulk pinning, the polar plot area increases as a function of the frequency.

To compare different superconductors and to clarify differences or similarity, we plot in Fig. 10 the initial $\Theta$ angle values vs. frequency for different HTSC materials. $\mathrm{NdFeAsO} \mathrm{O}_{1-0.14} \mathrm{~F}_{0.14}, \mathrm{YBCO}$ and $\mathrm{MgB}_{2}$ exhibit a similar frequency behavior suggesting the presence of a 3D flux-pinning behavior in contrast to the opposite 2D Bi2223 flux pinning response. Moreover it is also evident that different slopes and the presence or absence of a plateau in the curves vs. frequency address the presence of different collective weak pinning distributions inside these materials. In particular, while for the $\mathrm{YBCO}$ and $\mathrm{MgB}_{2}$ samples the frequency independent critical state at $1070 \mathrm{~Hz}$ is not yet achieved, at the same frequency YBCO the $\Theta$ value is $58^{\circ}$ while for the $\mathrm{MgB}_{2}$ we find $\Theta=74^{\circ}$. For the latter the ideal critical state $\left(\Theta=90^{\circ}\right)$ is compatible at high frequency addressing the better flux pinning characteristic of the $\mathrm{MgB}_{2}$ respect to the $\mathrm{NdFeAsO}_{1-0.14} \mathrm{~F}_{0.14}$ sample that exhibits a plateau already starting at low frequencies.

In the $\mathrm{NdFe} \mathrm{AsO}_{1-0.14} \mathrm{~F}_{0.14}$ in a 'real' critical state an independent frequency behavior is achieved. This suggests that this material with good 3D pinning characteristics is capable to sustain high critical currents. The different frequency slopes showed in Fig.10 are correlated to the different thermal activations and to the flux pinning dimensionality of the superconductors.

Finally, from the polar plot shapes in Fig. 7 both YBCO and $\mathrm{NdFeAsO} \mathrm{O}_{1-0.14} \mathrm{~F}_{0.14}$ samples show similar pinning structures. The $\mathrm{X}_{3}$ polar plots have ellipsoidal shapes and their different frequency behaviors depend by the different weight of the temperature activation respect to the pinning potential in the flux creep motion. Actually, for the YBCO sample the critical temperature is around $90 \mathrm{~K}$, while it is only $45 \mathrm{~K}$ for the $\mathrm{NdFeAsO}_{1 \text { - }}$ ${ }_{0.14} \mathrm{~F}_{0.14}$ sample.

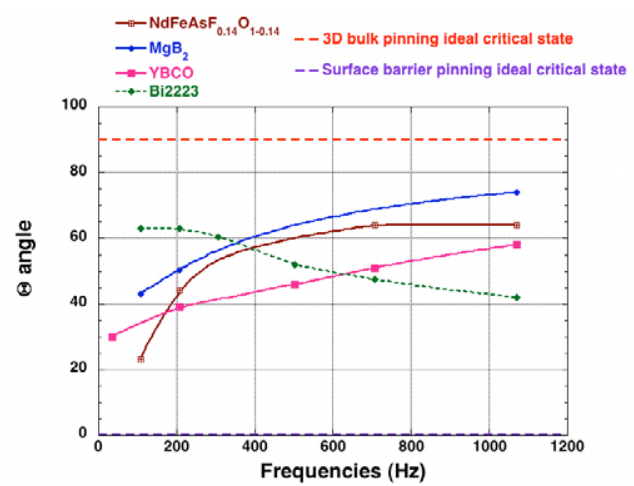

Fig. 10. The $\Theta$ angle vs. frequency for the $\mathrm{NdFeAsO}_{1-0.14} \mathrm{~F}_{0.14}$, $\mathrm{MgB}_{2}, \mathrm{YBCO}$ and Bi2223 samples. The $\Theta_{\text {critical state }}$ for bulk $\left(=90^{\circ}\right)$ and surface barrier pinning $(=0)$ is also showed. The $\Theta$ angle has been defined in the $\chi_{3}$ polar plot.

\section{V.CONCLUSIONS}

The third harmonic, Cole-Cole polar plots as a function of the frequency of the magnetic excitation may explore the complex flux-pinning dynamic response of a superconductor. In this contribution we demonstrate that a 3D weak collective bulk pinning is active in the $\mathrm{NdFeAsO}_{1-0.14} \mathrm{~F}_{0.14}$. In this framework, it is clear that the amplitude of the $\Theta$ angle and the shape of the $X_{3}$ polar plot define, respect to the experimental set-up considered, the flux-pinning interaction of a superconductor.

\section{ACKNOWLEDGMENTS}

A special thank is due to F. Tabacchioni (INAF) and to the entire technical staff of the DAФNE-Light laboratory for their continuous technical support.

\section{REFERENCES}

[1] Y. Kamihara, T. Watanabe, M. Hirano, and H. Hosono, J. Am. Chem. Soc. 130, 3296 (2008).

[2] D.J. Singh and M.H. Du, Phys. Rev. Lett 100, 237003 (2008).

[3] Y. Kohama,Y. Kamihara, M. Hirano, H. Kawayi, T. Atake and H. Hosono, Phys. Rev. B 78, 020512 (R) (2008).

[4] C. de la Crutz, Q. Huang, J.W. Lynn, J.Li, W. Ratcliff II, J.L. Zarestky, H.A. Mook, G.F. Chen, J.L. Luo, N.L. Wang \& P. Dai, Nature, 453, 899 (2008).

[5] C. Senatore, R. Flukinger, M. Cantoni, G. Wu, R.H. Liu and X.H. Chen, Phys. Rev. B 78, 054514 (2008)

[6] F. Hunte, J. Jaroszynski, A. Gurevith, D.C. Larbalestier, R. Jin, A.S. Sefat, M.A. McGuire, B.C. Sales, D.K. Christen and D. Mandrus, Nature 453, 903 (2008) 
[7] T. Ishida, R.B. Goldfarb, Phys. Rev. B 41 (1990) 8937.

[8] D. Di Gioacchino, P. Tripodi, F. Celani, A.M. Testa, S. Pace, Phys. Rev. B 59 (1999) 11539

[9] J.R. Clem, 'Magnetic Suscepttibility of Superconductors and Other Spin System', ed. in: T.L. Francavilla, D.H. Liebenberg, Plenum Press (1991), 177.

[10] C. Bean, Rev. Mod. Phys. (1964) 31

[11] Y.B. Kim, C.F. Hempstead, A.R. Strnad, Phys. Rev. 129 (1963) 528

[12] C.P. Poole, H.A. Farach, R.J. Creswick, Superconductivity Academic Press (1995) 365

[13] S. Shatz, A. Shaulov, Y. Yeshurun, Phys. Rev. B 48 (1993) 13871

[14] A.M. Campbell and J.E. Evetts, Adv. Phys 21 (1972) 199

[15] Y. Wolfus, Y. Abulafia, L. Klein, V.A. Larkin, A. Shaulov, Y. Yeshurun, M. Konczykowsi, M. Feigel'man, Physica C 224 (1994) 213-220

[16] G. Blatter, M.V. Feigel'man, V.B. Geshkenbein, A.I. Larkin, V.M. Vinokur, Rev. Mod. Phys. 66, 4 (1994) 1217

[17] D. Di Gioacchino, P. Tripodi, F. Celani, A.M. Testa, S. Pace, IEEE Trans. Appl. Sup. 11 (2001) 3924

[18] W. Yi, L. Sun, Z. Ren, W. Lu, X. Dong, H. Zhang, Xi Dai, Z. Fang, Z. Li, G. Che, J. Yang, X. Shen, F.Z.Z. Zhao, Europhys. Lett. 83, 57002 (2008)

[19] D. Di Gioacchino, U. Gambardella, P. Tripodi, G. Grimaldi, Sup. Sci. Tech. 16 (2003) 534.

[20] J. Gilchrist, T. Dombre, Phys. Rev. B 49 (1994) 1466

[21] C.J. van der Beek, M.V. Indenbom, G. D'Anna, W. Benoit, Physica C 258 (1996) 105-120

[22] D. Di Gioacchino, P. Tripodi, U. Gambardella, V. Sandu, S. Popa, L. Miu, D. Vinko, Physica C 408-410 (2004) 120

[23] M.J. Quin, C.K. Ong, Physica C 334 (2000) 107-114

[24] G. Blatter, M.V. Feigel'man, V.B Geshkenbein, A.I. Larkin, V.M. Vinokur, Rev. Mod. Phys. 66, 4 (1994) 1133

[25] G. Blatter, M.V. Feigel'man, V.B. Geshkenbein, A.I. Larkin, V.M. Vinokur, Rev. Mod. Phys. 66, 4 (1994) 1269

[26] G. Blatter, M.V. Feigel'man, V.B. Geshkenbein, A.I. Larkin, V.M. Vinokur, Rev. Mod. Phys. 66, 4 (1994) 1232

[27] A.I. Larkin and Y. Ovchinnikov, Jour. Low Temp. Phys. 34 (1979) 409

[28] E.H. Brandt, J. Low Temp. Phys. 53 (1983) 41

[29] L.F. Cohen and H.J. Jensen, Rep. Prog. Phys. 60 (1997) 1602

[30] A.M. Campbell and J.E. Evetts, Adv. Phys. 21 (1972) 199

[31] G. Blatter, M.V. Feigel'man, V.B. Geshkenbein, A.I. Larkin, V.M. Vinokur, Rev. Mod. Phys. 66, 4 (1994) 1314 